\title{
Acute Post-Streptococcal Glomerulonephritis in Children - A Review
}

\author{
RANJIT RANJAN ROY ${ }^{1}$, KAMRUL LAILA $^{2}$
}

\begin{abstract}
Acute poststreptococcal glomerulonephritis (APSGN) is characterized by abrupt onset of hematuria, edema, hypertension, oliguria and impaired renal function following streptococcal group A â hemolytic streptococcal throat and skin infection. There is a declining incidence of APSGN worldwide, particularly in industrialized nations because of easier and earlier access to competent medical treatment of streptococcal infections and the widespread use of fluorination of water since virulence factors in streptococcus pyogens are reduced with fluoride exposure. But in the underdeveloped world, global burden of APSGN continues to be significant with lower estimate of 9.3 to 9.8 cases per 1,00,000 population per year to higher estimates as high as three times these values. Furthermore, clusters of cases are more frequently reported in poor communities in industrialized countries while epidemics of more than 100 cases are reported in the middle ranger countries with mean annual health expenditure per capita of about 550 US dollars.
\end{abstract}

APSGN typically follows 1 to 2 weeks after pharyngeal infection and 2 to 4 weeks after skin infection by nephritogenic strains of group $A$ â hemolytic streptococcus in a range of 5-15 years of age. Subclinical cases are 4-10 times higher than symptomatic patients. The acute phase generally resolves within 4-8 weeks but microscopic hematuria may persist for 1-2 yr after the initial presentation.

Acute complications of symptomatic patients are hypertensive heart failure, encephalopathy and retinopathy. There can be acute renal failure and rarely rapidly progressive ( crescentic) glomerulonephritis, hyperkalemia, hyperphosphatemia, hypocalcemia and acidosis.

Treatment is directed towards reduction of hypertension, but prompt address of complications are essential to avoid immediate mortality. Heart failure is treated with diuretic and anti-hypertensive, digoxin is ineffective. Hypertensive encephalopathy is treated by I.V phenobarbitone for convulsion, supportive measures for unconsciousness and blood pressure control. Acute renal failure is managed by supportive measures, rarely requires dialysis. Short and long term prognosis is excellent, with1\% mortality during acute stage and 1\% ending up with chronic kidney disease, but in higher age group abnormal urinalysis are present in higher number of patients.

\section{Introduction:}

Acute poststreptococcal glomerulonephritis (APSGN) is characterized by abrupt onset of hematuria, edema, hypertension, oliguria and diminished renal function after group Aâ hemolytic streptococcus infection. ${ }^{1}$ In paediatric age group acute poststreptococcal

1. Associate Professor, Paediatric Nephrology, Bangabandhu Sheikh Mujib Medical University, Shahbag, Dhaka

2. Resident, Paediatrics, Bangabandhu Sheikh Mujib Medical University, Shahbag, Dhaka

Correspondence: Dr. Ranjit Ranjan Roy, Associate Professor, Paediatric Nephrology, Bangabandhu Sheikh Mujib Medical University, Shahbag, Dhaka, Cell : 01715-004079, E.mail: ranjit.bsmmu@gmail.com glomerulonephritis (APSGN), accounts for approximately $80 \%$ of cases of acute nephritic syndrome (ANS). ${ }^{1,2}$

The disease is now rare in industrialized nations, but in the unprivileged world, the number of APSGN ranges between 9.5 and 28.5 new cases per year. ${ }^{3}$ In more developed countries, the incidence is estimated at 0.3 cases per $1,00,000$ children year on the basis of the Italian biopsy registry. ${ }^{4}$ Infection associated nephritis has practically disappeared in central Europe where it is now frequent in elderly people specially in association with debilitating conditions such as alcoholism and intravenous drug abuse. ${ }^{4}$ In China and 
Singapore, a reduction in the incidence of APSGN has been noted in past 40 years. APSGN remains a common disease in many rural and aboriginal communities with low socioeconomic status such in the cases in Australia, Valencia, Venezuela where the disease causes $70 \%$ of hospital admission in a paediatric nephrology service.$^{4}$ In developing country like India, APSGN represents $73 \%$ of AGN affecting elderly which may or may not represent a shift in age predominance such as has been referred to previously for central Europe. ${ }^{4,5}$ Epidemic outbreak tend to occur in closed populations such as red lake Indian reservations in Mennesota, USA and less developed countries as Port of Spain in Trinidad, Maracaibo, Venezuela. The risk of nephritis in epidemic is $5 \%$ in throat infection, to as high as $25 \%$ in pyoderma ${ }^{4}$. Overall risk with nephritogenic strains is about 15 percent. $^{1}$

In Bangabandhu sheikh Mujib Medical University (BSMMU), Dhaka, Bangladesh, in the year of 2013,among a total number of 3636 patients attending the paediatric nephrology outpatient department, 98 patients were diagnosed as APSGN which was 2.76 $\%$ of the total. Among the 577 admitted in paediatric nephrology department of BSMMU in 2013, there were 35 cases (6\%) of APSGN. And among these 35 cases, 5 patients (14.2\%) developed acute renal failure, 2 patients $(5.7 \%)$ developed hypertensive encephalopathy and another 2 ( $5.7 \%)$ had hypertensive heart failure.

\section{Etiopathogenesis:}

APSGN typically follows 1 to 2 weeks after pharyngeal infection and 2 to 4 weeks after skin infection caused by nephritogenic strains of group A â hemolytic streptococcus. ${ }^{1}$ Serotypes implicated in pharyngeal and skin infections are $M$ type 1,3,4,12,25,49 and $M$ type $2,49,55,57,60$ respectively; 12 and 49 are the commonest. ${ }^{1}$ However, more recently it has been found that glomerulonephritis may result from group C streptococci, as noted earlier making thus evident that antigenic fractions capable of causing nephritis are shared by a large variety of streptococci. Potential nephritogenic antigens include $M$ protein, endostreptosin (preabsorbing) antigen, cationic protein, nephritis strain associated protein, streptococcal pyrogenic exotoxin B(SPEB) and nephritis associated plasmin receptor(Naplr) ${ }^{1}$. Naplr deposits are present in early biopsies and Naplr antibody levels are detected by western blot in convalescent sera. ${ }^{1}$
Host factor like 2:1 male female ratio, tropical climate, lower socioeconomic background, crowded living conditions, poor hygiene, malnutrition, anemia, parasitic infestation have all been implicated as predisposing factors for APSGN. ${ }^{2}$ Genetic risk factors for example HLA DRW4, HLADPA1 and HLADPB alleles are more prevalent in patients with APSGN than normal population. ${ }^{2}$ Sporadic disease is common but epidemic outbreaks tend to occur in closed populations and in less developed countries. ${ }^{6,7}$

$\mathrm{M}$ protein contributes to virulence and glomerular injury. ${ }^{2}$ Streptokinase involved in spread of bacterium through tissue. ${ }^{2}$ Two antigens are actively investigated at the present time as the potential causes of APSGN: the nephritis associated plasmin receptor (NAPIR),identified as glyceraldehydes 3 phosphate dehydrogenase and a cationic cysteine proteinase known as streptococcal pyrogenic exotoxin B(SPEB) are capable of activating the alternate pathway of complement activation. ${ }^{8}$

Molecular mimicry between streptococcal antigens and glomerular antigens that react with antibodies against streotococcal antigens and direct activation of complement cascade by both classical and alternate pathways occur. ${ }^{1}$ Infiltration of neutrophil, proliferation of glomerular cells, mesengial cell and expansion of mesengial matrix take place(type iii immune complex reaction $)^{1}$,glomeruli are diffusely enlarged and lobular pattern is accentuated. ${ }^{1,8}$

In a $0.5 \%$ of cases parital epithelial cell proliferation in cresentic manner are present which are associated with a rapidly progressive course (RPGN) and a poor prognosis ${ }^{1,2,8}$, interstitial edema is also noted. Cresents compress the underlying glomeruli making them non functional and hence loss of nephrons. In cresentic glomerulonephritis more than $50 \%$ glomeruli are involved. . $^{1,2,8}$

Immunofluorence examination shows irregular granular deposits of IgG and C3 along capillary walls and mesangium, fine granular deposits of $\mathrm{C} 3$ and IgG along capillary wall is labeled as "starry sky" appearance and noted in early phase of disease. ${ }^{1,8} \mathrm{~A}$ garland pattern of dense confluent deposits sparing the subendothelial and mesengial location is correlated with heavy proteinuria. ${ }^{1,8}$

APSGN has an immune complex pathogenesis. Immune complexes formed against nephritogenic streptococcal antigen(s) may be formed in circulation, 
and deposited in the glomeruli, or formed in situ against antigenic fractions planted in the glomeruli. ${ }^{4}$ The relevance

of the circulating immune complex mechanism derives from the classic experiments of the glomerulonephritis in acute serum sickness; however, glomerular deposition of free antigen and in-situ immune complex formation has been more recently proposed as an important pathogenetic mechanism in APSGN. Such a mechanism would easily explain the formation of subepithelial electron dense deposits (humps) which are extremely difficult to produce by the injection of preformed immune complexes and are, in contrast, the rule with the injection of cationic antigens. Since the existence of a large number of humps in biopsies is associated with heavy proteinuria and worse prognosis, the in-situ mechanism may have significant clinical relevance. APSGN results from the inflammatory reactivity induced by the immune complexes involving complement activation. Complement activation occurs preferentially by the alternate pathway. Ig-binding proteins in streptococcal surface interfere with the classical pathway of complement activation. Recent studies suggest that complement regulatory proteins that play a role in protecting streptococci may be removed by SPEB. Recent investigations have shown that the lectin pathway of complementmay also be activated in APSGN by the recognition of glucosamine residues. ${ }^{4}$

In addition to humoral immunity, cell-mediated mechanisms are also involved in the development of APSGN. ${ }^{4}$ Glomerular infiltration of lymphocytes and macrophages has long been recognized as a feature of the disease. Intercellular leukocyte adhesion molecules, such as ICAM-1 and leucocyte function assisted antigen (LFA) are over expressed in glomeruli and tubulointerstitial regions and correlated with the intensity of the inflammatory infiltration. ${ }^{4}$

A number of autoimmune findings have been reported in APSGN. Among them, anti-IgG reactivity is the best studied. ${ }^{4}$ Cryoglobulins and high titers of rheumatoid factor are present in about two-third of the patients in the first week of the disease; furthermore, anti-IgG glomerular deposits are frequently found in biopsies (see later) and anti-IgG reactivity has been documented

in the IgG eluted from the kidney in a fatal case of the disease. The anti-lgG reactivity may be the result of autoantigenic modification of Ig that occurs with its desialization caused by streptococcal neuraminidase. This possibility was raised by experimental studies showing that injection of neuraminidase-treated autologus IgG causes the appearance of anti-IgG reactivity and received support from the demonstration of plasma neuraminidase activity and increased free sialic acid levels in patients with APSGN. Another potential cause of anti-Ig reactivity is the binding of IgG to type II receptors in the streptococcal wall. AntiIgG reactivity is a constant feature of injections of streptococci cultured in a medium with autologous serum.

Additional autoimmune phenomena have been found in APSGN patients. Anti-DNA antibodies, antiCiq antibodies and anti-neutrophil cytoplasmic antibodies (ANCA) are present in some patients. Interestingly, the later has been found in two-third of the patients with azotemia and $70 \%$ of the patients with APSGN that develop crescentic glomerulonephritis. ${ }^{4}$

The pathogenetic importance of cellular immune mechanisms was suggested by early studies that demonstrated lymphocyte and macrophage infiltration in renal biopsies of patients with the disease. ${ }^{4}$ The infiltration of immunocompetent cells is facilitated by overexpression of intercellular adhesion molecules (ICAM-1) and LFA-1 in APSGN. Cytokine and chemokine production is a central process in damage induced by immune cells. Interleukin-6 (IL-6) plays an important role in proliferative glomerulonephritis and one of the putative nephritogenic antigens, SPEB, induces increased production of IL-6 and proliferation on mesangial cells. Glomerular IL-8 correlates with neutrophil infiltration and transforming growth factorâ with mesangial expansion and there is a direct correlation between serum tumor necrosis factor (TNFá) levels and glomerular ICAM-1 expression. ${ }^{4}$

\section{Clinical presentation of APSGN:}

Children from 4 to 14 years are more frequently affected by APSGN. It is rare below the age of 2 and above the age of 20 and is twice more frequent in males than in females. ${ }^{4}$ In most cases there is a history of sore throat or pyoderma 1-2 weeks and 2-4 weeks respectively ${ }^{1,2,8}$. Typically patient present with hematuria in $100 \%$ cases, $30-70 \%$ of whom have gross hematuria. ${ }^{1,2,8}$. ASPGN may have subclinical course or may present with the acute nephritic syndrome, and more rarely with nephrotic syndrome(4 - $10 \%$ ) or, exceptionally, with a rapidly 
progressive(crescentic) glomerulonephritis $(0.5 \%)^{9}$. Cervical lymphadenopathy may be the residua of a recent streptococcal pharyngitis. ${ }^{10}$

Subclinical disease is characterized by a reduction of serum complement, microscopic hematuria and normal or increased blood pressure in asymptomatic patients. Prospective studies in household members of index cases have shown that in non-epidemic situations the patients without symptoms are 4-10 times more frequent than symptomatic patients ${ }^{11,12}$.

The most typical clinical picture in APSGN is the acute nephritic syndrome (hematuria, edema, hypertension and mild azotemia). The history begins with obtaining more details about the change in urine. Hematuria in children with AGN is typically described as "coke," "tea," or "smoky" colored. True bright red blood in the urine is more likely a consequence of anatomic problems such as urolithiasis ${ }^{13}$ than glomerulonephritis. ${ }^{14}$ Glomerular hematuria is an almost universal finding and gross hematuria is present in one-third of the patients. The absence of red cells in the urine is usually due to delays in the examination of the urinary sediment since red cells are rapidly destroyed, especially in alkaline urine. As a rule, red cell casts are present in association with dysmorphic red cells, frequently presenting doughnut shape with one or more blebs. ${ }^{4}$ Macroscopic hematuria usually disappears after a few days but microscopic hematuria may persist for a year and occasionally exacerbates during febrile episodes and more rarely after strenuous exercise. $^{4}$

Edema is the chief complaint more frequently in children (90\%) than in adult patients (75\%). ${ }^{4}$ Edema typically results from salt and water retention and nephrotic syndrome may develop in 4-10\% of cases, ${ }^{7}$ but this tends to be a more subtle "brawny" edema than the pitting edema characteristic of nephrotic syndrome. Oliguria is referred on admission by the patient or their family in less than half of the patients. ${ }^{7}$

Hypertension is present in $60-80 \%$ of the children and is severe enough to require specific antihypertensive treatment in about half of the cases. ${ }^{4}$ Sometimes, complications of high blood pressure such as headache, vomiting, dizziness or seizures that bring attention to the presence of acute poststreptococcal glomerulonephritis. ${ }^{15}$

Patients may develop encephalopathy owing to hypertension or hypervolemia which is manifested by headache and convulsion. ${ }^{1}$ Encephalopathy may also result directly from the toxic effects of the streptococcal bacteria on the central nervous system. Five percent patients of APSGN may develop heart failure as a complication of hypertension - initial left ventricular failure, followed by right heart failure and pulmonary edema simulating pneumonia or myocarditis. ${ }^{1}$

Some nonspecific symptoms such as malaise, lethargy, abdominal or flank pain, and fever are common. Acute subglottic edema and airway compromise has also been reported. ${ }^{7}$

Azotemia occurs in $25-30 \%$ of the patients but the need of dialysis is infrequent. A rapidly progressive azotemia occurs in less than $0.5 \%$ of the cases and when present is due to the development of crescentic glomerulonephritis, also called rapidly progressive glomerulonephritis (RPGN) ${ }^{4}$ which must be intervened quickly otherwise there can be immediate mortality and long term sequelae. Massive proteinuria with or without other features of the nephrotic syndrome are found in about $4-10 \%$ of the cases and its persistence is a risk factor for progression to chronic renal disease. ${ }^{4}$

Edema and hypertension typically disappear in 5-10 days. ${ }^{4}$ The high blood pressure, protein and blood in the urine eventually go away and the C3 eventually returns to normal in about 2 months time. ${ }^{15}$

The acute phase generally resolves within $6-8 \mathrm{wk}^{7}$ Although urinary protein excretion and hypertension usually normalize by 4-6 wk after onset, persistent microscopic hematuria may persist for 1-2 yr after the initial presentation. ${ }^{7}$

\section{Conditions that mimic APSGN $1,2,4$ :}

- Other post infectious AGN-bacterial, viral, fungal, rickettsial, parasitic etc.

- Vasculitis - Systemic lupus erythematosus, Henoch schonlene purpura, Wegeners granulomatosis, microscopic polyangitis.

- IgA nephropathy

- Acute on chronic glomerulonephritis - commonly membranoproliferative glomerulonephritis, mesangioproliferative glomerulonephritis.

- Alports syndrome

- Acute interstitial nephritis

\section{Complications:}

Acute complications of this disease result primarily from hypertension and acute renal dysfunction. ${ }^{5}$ APSGN may develop heart failure as a complication 
of hypertension - initially left heart failure followed by right heart failure and pulmonary edema simulating pneumonia or myocarditis.

Patients may develop encephalopathy owing to hypertension or hypervolemia which is manifested by headache and convulsion. Encephalopathy may also result directly from the toxic effect of streptococcus on central nervous system. ${ }^{5}$ Another potential complication, frequently associated with hypertension is the posterior reversible leukoencephalopathy that has recently been reported in acute PSGN. ${ }^{16}$ This complication is manifested clinically with mental disturbances, visual hallucinations, headache and convulsions and may be confused with hypertensive encephalopathy. The diagnosis requires the use of nuclear magnetic resonance image studies. ${ }^{4}$

Other potential complications include rapidly progressive glomerulonephritis, hyperkalemia, hyperphosphatemia, hypocalcemia, acidosis. ${ }^{4,5}$ One percent patient develop chronic kidney disease in the long run. ${ }^{4,5}$

\section{Conditions that mimic APSGN ${ }^{1,2,4}$ :}

- Other post infectious AGN-bacterial, viral, fungal, rickettsial, parasitic etc.

- Vasculitis - Systemic lupus erythematosus, Henoch schonlene purpura, Wegeners granulomatosis, microscopic polyangitis.

- IgA nephropathy

- Acute on chronic glomerulonephritis - commonly membranoproliferative glomerulonephritis, mesangioproliferative glomerulonephritis.

- Alports syndrome

- Acute interstitial nephritis

\section{Diagnosis}

Urinalysis demonstrates dysmorphic red blood cells (RBCs), frequently in association with RBC casts, pus cell (does not mean urinary tract infection) ${ }^{1}$ and proteinuria. Complete blood count shows polymorphonuclear leukocytosis. A mild normochromic anemia may be present from hemodilution and lowgrade hemolysis. ${ }^{5}$

They may also have increased levels of serum creatinine, also there may be rise in blood urea and blood urea nitrogen ${ }^{22}$. Serum electrolyte analysis may reveal hyponatremia, hyperkalemia, hypocalcemia, hyperphosphatemia and metabolic acidosis. ${ }^{4}$
The most constant serological finding is the reduction in serum complement levels that occurs in more than $90 \%$ of the cases. ${ }^{4}$ The serum C3 level is usually reduced in the acute phase and returns to normal 6-8 wk after onset. ${ }^{7}$

A positive throat culture report may support the diagnosis or may simply represent the carrier state. ${ }^{1}$ On the other hand, a rising antibody titer to streptococcal antigen confirms a recent streptococcal infection. ${ }^{1}$ Rising antistreptococcal antibody titers are the usual clinical indication of a preceding streptococcal infection since positive cultures are obtained in only $20-25 \%$ of the cases, except during epidemics. ${ }^{6}$ It starts rising within 2 weeks, peaks at 4 weeks and falls at 12 weeks. ${ }^{1}$ Importantly, the antistreptolysin $\mathrm{O}$ titer is commonly elevated after a pharyngeal infection but rarely increases after streptococcal skin infections because of the presence of thick lipid barrier of skin. The best single antibody titer to document cutaneous streptococcal infection is the deoxyribonuclease (DNase) B antigen. The Streptozyme test (Wampole Laboratories, Stamford, $\mathrm{CT}$ ) is an alternative study that detects antibodies to streptolysin O, DNase B, hyaluronidase, streptokinase, and nicotinamide-adenine dinucleotidase using a slide agglutination test. ${ }^{2,7} \mathrm{Anti}-$ zSPEB/SPEB (Streptococcal pyogenic exotoxin B and its zymogen precursor) serum titers have been found to be the best markers of nephritogenic streptococcal infections in multicentric studies in LatinAmerica. ${ }^{17,18}$ Naplr antibody is a measures of protection, found raised in $92 \%$ of patient. $4,19,20$.

In first weeks of disease two-third of the APSGN patient may have cryoglobins and elevated IgG-anti-lgG rheumatoid factor titres. ${ }^{21}$

Diagnosis of skin infection is straightfroward clinically but half of the phyryngitis may be missed and 20$40 \%$ missdiagnosed. ${ }^{22}$ So for accuracy Mclssac et al proposed a clinical score ranging from 0-4. Tempareture $>38^{\circ} \mathrm{C}$, cervical lymphadenopathy, no cough, tonsillar exudate and age 3 to 14 years are given score +1 and -1 for age $>44$ years. ${ }^{4,23}$ Sensitivity and specificity of the score is 85 and $95 \%$, respectively. Antibiotic treatment is recommended (without culture confirmation) when the score is 4 , and antibiotic treatment is not indicated (and cultures are unnecessary) when scores are $0-1$, in case of score 1-3 clinical examination and culture sensitivity are considered. ${ }^{6}$ IgG and IgM serum levels are elevated 
80-90\% of the patients with APSGN. Confirmation of the diagnosis requires clear evidence of invasive streptococcal infection. ${ }^{6}$

\section{Indication of renal biopsy}

Renal biopsy is not required in most of APSGN but it is indicated when there is delayed resolution and complication and also to exclude some differential diagnosis. Followings are the indications. ${ }^{5}$

- gross hematuria for more than 1 month

- microscopic hematuria for more than 1 year

- hypertension, oliguria, azotemia for more than 2 weeks

- nephrotic range proteinuria for more than 2 weks

- persistent proteinuria for more than 6 months.

- Suspected vasculitis, IgA nephropathy, Alport syndrome, acute and chronic glomerulonephritis, Rapidly progressive glomerulonephritis(RPGN), renal biopsy should be done. ${ }^{1,5}$

\section{Treatment}

Management is directed at treating the acute effects of renal insufficiency and hypertension. ${ }^{4}$ Patients with subclinical disease may be followed as outpatients but patients with the acute nephritic syndrome with severe hypertension and complication require hospitalization. ${ }^{1,4}$

Bed rest is difficult to enforce and is of unproven value, yet most children keep it on their own while they are in the acute phase ${ }^{4}$. Restrictions of fluid and sodium intake are the cornerstones of the treatment of patients with APSGN. ${ }^{4}$

Cases that present significant edema, hypertension and circulatory congestion benefit from the administration of loop diuretics (1-2 mg/kg, maximum upto $10 \mathrm{mg} / \mathrm{kg}$ IV or orally every $12 \mathrm{~h}$ ). This therapy facilitates the resolution of edema and ameliorates the hypertension that is driven by extracellular volume expansion. Diuretic therapy seldom if ever is required for longer than $48 \mathrm{~h}$. Other diuretics are without effect (thiazide diuretics) or dangerous because of the possibility of hyperkalemia (aldosterone antagonists). ${ }^{4}$

Patients who present severe hypertension may require antihypertensive treatment and Nifedipine $(0.5-2 \mathrm{mg} /$ $\mathrm{kg}$ in children, every $4-6 \mathrm{~h}$ ) is usually effective. Sublingual Nifedipine and intravenous Nicardipin can also be used. Beta blockers are usually avoided as patients develop some degree of respiratory compromise except Metoprolol which is a super selective beta blocker. Parenteral hydralazine, Labetelol, Diazoxide may be required for hypertensive emergency but the possibility of tachycardia requires close observation. Angiotensin converting enzyme inhibitors and type 1 receptor blockers carry the risk of hyperkalemia and should not be used when GFR is $<30 \%$ and serum potassium is $>5.8 \mathrm{mmol} / \mathrm{L}$. Exceptionally, nitroprusside is required to control hypertensive encephalopathy. ${ }^{4}$

Complications of $A G N$ should be treated simultaneously ${ }^{1}$. For RPGN intravenous methyl prednisolone along with cyclophosphamide can be considered followed by oral prednisolone and dialysis if necessary. Other immunosuppressive drugs like azathioprim mycophenolate mofetil are also used. 1,4 Diuretics and control of hypertension in heart failure, dialysis and other supportive measures for acute renal insufficiency, symptomatic management for encephalopathy eg. IV phenobarbitone for convulsion, nebulized salbutamol and others for hyperkalemia, Intravenous sodibicarb for acidosis should be kept in mind. ${ }^{1}$

There is no specific treatment for post-streptococcal glomerulonephritis. Treatment is focused on relieving symptoms. ${ }^{24}$ The first question to be considered is when to give antibiotic treatment to a suspected nephritogenic streptococcal infection. ${ }^{4}$ Rapid, high sensitivity streptococcal test are good guide to treat if they are positive but a negative test requires confirmation ${ }^{25}$. However, a recent report indicates that a decision to treat or not to treat based on the results of these tests is not associated with a higher incidence of poststreptococcal AGN after sore throat and skin infection ${ }^{26}$. The diagnosis of PSGN carries with it the indication of treatment with penicillin or, in allergic individuals, erythromycin. If infection is present at the time of diagnosis, it requires treatment. Early administration of penicillin is reported to prevent or ameliorate the severity of acute glomerulonephritis and at least one report suggests that APSGN patients that receive antibiotic treatment have a milder clinical course. ${ }^{27,28}$ If infection is not apparent at the time of diagnosis, antibiotic treatment should be given anyway because positive cultures are sometimes obtained in apparently healthy patients and cross infection of household members and siblings of index cases is very high. ${ }^{12}$ 
Although a 10-day course of oral antibiotic therapy with penicillin is recommended to limit the spread of the nephritogenic organisms, antibiotic therapy does not affect the natural history of glomerulonephritis. ${ }^{5}$ Corticosteroids and other anti-inflammatory medications are generally not effective. ${ }^{24}$

\section{Prognosis of APSGN}

The short term prognosis of APSGN is excellent in children ${ }^{1}$. 99\% recovery within weeks, less than $1 \%$ has immediate mortality. ${ }^{1}$ Fatalities may occur as a result of hyperkalemia or pulmonary edema. The long term prognosis of APSGN has been the subject of many reports since the initial studies in the first half of the twentieth century reported essentially a complete recovery, but the follow-up periods were short and the patients were only children in the majority of the studies. ${ }^{4}$

APSGN followed for 10-20 years present frequently abnormal urine analysis (20\%) but azotemia occurs in less than $1 \%$ of the patients. ${ }^{1}$ But in adult prognosis is worse with incidence of persistent proteinuria and chronic kidney disease after 5 years in $8 \%$ of this patient. ${ }^{29,30}$

Mortality in the acute stage can be avoided by promt and appropriate management of acute renal failure, cardiac failure, and hypertension. Infrequently, the acute phase may be severe and lead to glomerular hyalinization and chronic renal insufficiency ${ }^{5}$. However, the diagnosis of acute poststreptococcal glomerulonephritis must be questioned in patients with chronic renal dysfunction because other diagnoses such as membranoproliferative glomerulonephritis may be present. Recurrences are extremely rare(0.7-1\%) because of limited number of nephritogenic strain and protective antibody. ${ }^{5}$

Occasionally, glomerulonephritis may remain silent for a long period until kidney failure occurs. Symptoms of kidney failure include lack of appetite, nausea and vomiting, fatigue, difficulty sleeping, dry and itchy skin, and passing a smaller amount of urine than normal. ${ }^{5}$ Glomerulonephritis can be sudden and severe progressing to kidney failure very rapidly — but this is rare. ${ }^{30}$ Based on long term experience less than $2 \%$ children with this disease need dialysis or kidney transplant. ${ }^{15}$ So, follow up of a patient of APSGN should include urinalysis, serum creatinine, measurement of blood pressure, anemia and anthropometry for $1-2$ years. ${ }^{1}$

\section{Prevention}

Early systemic antibiotic therapy for streptococcal throat and skin infections does not eliminate the risk of glomerulonephritis. ${ }^{5}$ Family members of patients with acute glomerulonephritis should be cultured for group Aâ-hemolytic streptococci and treated if culture positive. ${ }^{5}$

\section{Conclusion:}

Despite global decline of APSGN, still it is a health problem in less wealthy nation. Prompt address of hypertension and complications are the main stay of treatment. Immediate outcome is excellent at expert hand and $1 \%$ can develop chronic kidney disease in long-term. Recurrence is very rare $(<1 \%)$.

\section{References}

1. Bagga A, Srivastava RN, Acute and rapidly progressive glomerulonephritis in Paediatric Nephrology $5^{\text {th }}$ edn. Srivastava RN, Bagga A. Jaypee Brothers Medical publishers (P) Ltd. New Delhi 110002, India 2011, 130-150.

2. Smith JM, Faijan MK, Eddy AA, The child with nephritic syndrome. In clinical paediatric nephrology $3^{\text {rd }}$ edn. Webb $\mathrm{N}$ and Postlethwaite R. Oxford university press. New York 2003. 369379.

3. Iurbo BR, Musser JM. The current state of poststreptococcal glomerulonephritis. J Am Soc Nephrol 19; 1855- 1864, 2008.

4. Itube BR, Mezzano S. Acute post infectious glomerulonephritis. In Paediatric Nephrology. $6^{\text {th }}$ edn, edited by Avner ED, Harmon WE, Niaudet P, Yaskikawa N. Springer - Verlag Berlis Heidelberg, Germany, 2009. 743-755.

5. Rakesh J, Saxena RR, Sharma OP: Spectrum of renal disease in the elderly: single centre experience from a developing country. Int Uro. Nephrol 2001, 33 : 227-233.

6. Pan CG, Avner ED. Acute poststreptococcal glomerulonephritis. In Nelson Textbook of Paediatrics, $19^{\text {th }}$ edn. Edited by Kliegman RM, Stanton BF, St Game III JW, Schor NF, Berman RE. Elsevier Saunders. Philadelphia Pa 191032899, 2011. 1783-1794.

7. Rodriguez- Hurbo B, Batsford S: Pathogenesis of poststreptococcal glomerulonephritis : a century after clemons von Pirquet. Kidney Int 71, 2007: $1094-1104$. 
8. Jerkins GR Noe HN. Familial vesicoureteral reflux: a prospective study. J Urol 1982;128(4):774-778.

9. Eustaquio M E, Chan K H, Deterding R R, and Hollister R J, "Multilevel airway involvement in children with Wegener's granulomatosis: clinical course and the utility of a multidisciplinary approach," Archives of Otolaryngology-Head and Neck Surgery, 2011, 137( 5): 480-485.

10. Van den Abbeele AD et al. Vesicoureteral reflux in asymptomatic siblings of patients with known reflux: radionuclide cystography.Paediatrics 1987;79(1):147-153.

11. Noe HN. The long-term results of prospective sibling reflux screening. J Urol 1992;148(5 Pt 2):1739-1742.

12. Bourquia $A, Z$ aid $D$, Acute renal insufficiency in children: a retrospective study of 89 cases. Ann Pediatr, 1993, 40; 603-608.

13. B. Hoppe and M. J. Kemper, "Diagnostic examination of the child with urolithiasis or nephrocalcinosis," Paediatric Nephrology,2010: 25( 3); 403-413.

14. Ahn SY, Ingulli E. Acute poststreptococcal glomerulonephritis: an update. Curr Opin Pediatr 2008;20:157-162.

15. Iturbe B and Musser JM, The current state of post streptococcal glomerulonephritis, J Am Soc Nephrol,2008: 19; 1855- 1864.

16. Parra G, Rodrý'guez-Iturbe $B$, Batsford $S$ et al. Antibody to streptococca zymogen in the serum of patients with acute glomerulonephritis: a multicentric study. Kidney Int 1998; 54: 509-517.

17. Rodriguez-Iturbe B. Epidemic poststreptococcal glomerulonephritis(Nephrology Forum). Kidney Int 1984;25:129-136.

18. Cebul DR, Poses RM. The comparative costeffectiveness of statistical decision rules and experienced physicians in pharyngitis management. J Am Med Assoc 1986;256:33533357.

19. Oda T, Yamakami K, Omasu F et al. Glomerular plasmin-like activity in relation to nephritisassociated plasmin receptor in acute poststreptococcal glomerulonephritis. J

20. Yoshizawa N, Yamakami K, Fujino $\mathrm{M}$ et al. Nephritis-associated plasmin receptor and acute poststreptococcal glomerulonephritis: charac- terization of the antigen and associated immune response. J Amer Soc Nephrol 2004;15:17851793.

21. American Academy of Paediatrics, Committee on Infectious Diseases: Group A streptococcal infections. In The Red Book. Elk Groove Village, II. American Academy of Paediatrics 2000, 526592.

22. Webb KH, Needham CA, Kurtz SR. Use of a high-sensitivity rapidstrep test without culture confirmation of negative results: 2 years'experience. J Fam Prac 2000;49:34-36.

23. Mclsaac WJ,White D, Tannenbaum D et al. A clinical score to reduce unnecessary antibiotic use in patients with sore throat. Can Med Assoc J 1998;158:75-83.

24. Cotran, Ramzi S.; Kumar, Vinay; Fausto, Nelson; Nelso Fausto; Robbins, Stanley L.; Abbas, Abul K. (2005). Robbins and Cotran pathologic basis of disease. Edited by St. Louis, MO: Elsevier Saunders. pp. pp976-8.

25. Colville D, Guymer R, Sinclair RA, Savige J (August 2003). "Visual impairment caused by retinal abnormalities in mesangiocapillary (membranoproliferative) glomerulonephritis type II ("dense deposit disease")". Am. J. Kidney Dis. 42 (2): E2-5. doi:10.1016/S0272-6386(03)006656. PMID 12900843.

26. Habib R, Gubler MC, Loirat C, Mäiz HB, Levy M (1975). "Dense deposit disease: a variant of membranoproliferative glomerulonephritis". Kidney Int. 7 (4): 204-15.

27. Ronco P, Debiec H (2012) Pathogenesis of membranous nephropathy: recent advances and future challenges. Nat Rev Nephrol 2012, 35; 112115.

28. Ziakas PD, Giannouli S, Psimenou E, Nakopoulou L, Voulgarelis M (July 2004). "Membranous glomerulonephritis in chronic lymphocytic leukemia". Am. J. Hematol. 2004; 76 (3): 271-4.

29. Chugh KS, Sakhuja V, Baliga R. Glomerular disease in the tropics. Am J Nephrol 1990; 10: 437-450.

30. Sesso R, Pito SWL. Five years follow-up of patients with epidemic glomerulonephritis due to streptococcus zooepidermicus. Nephrol Dial Transplant 2005;20:1808-1813. 